\title{
An NMR Investigation of Phase Structure and Chain Dynamics in the Polyethylene/Montmorillonite Nanocomposites
}

\author{
Wei Li, Linxi Hou, and Zhongren Chen \\ Faculty of Material and Chemical Engineering, Ningbo University, Ningbo 315211, China \\ Correspondence should be addressed to Wei Li; liwei@nbu.edu.cn and Linxi Hou; houlinxi@nbu.edu.cn
}

Received 14 December 2012; Accepted 26 December 2012

Academic Editor: Jun Zhang

Copyright (C) 2013 Wei Li et al. This is an open access article distributed under the Creative Commons Attribution License, which permits unrestricted use, distribution, and reproduction in any medium, provided the original work is properly cited.

\begin{abstract}
Novel exfoliated and interacted polyethylene (PE)/montmorillonite (MMT) nanocomposites prepared by in situ polymerization were characterized by solid-state nuclear magnetic resonance (NMR). The phase structure and molecular mobility were investigated by proton and carbon NMR under static and magic-angle spinning (MAS) conditions. The results showed that incorporation of MMT layer enhanced the polyethylene crystallinity behavior. The chain mobility of crystalline phase, interphase and amorphous phase was hindered in the nanocomposites. The phase structure and chain dynamics were also investigated upon changing the temperature. The orthorhombic and monoclinic phases were detected according to the ${ }^{13} \mathrm{CP} / \mathrm{MAS}$ NMR. Quantitative characterization of the phase structure was also conducted by ${ }^{13} \mathrm{C} \mathrm{DP} / \mathrm{MAS}$ upon changing the temperature. Finally, the difference in the phase structure and chain dynamics in each phase of PE/nanocomposites was compared based on the NMR results when fiber filler was introduced.
\end{abstract}

\section{Introduction}

Polymer nanocomposites are a new class of particle filled polymers in which at least one composite consists of dispersed particles in the nanometer size range [1]. The layered silicate clay, such as montmorillonite (MMT), has been used in polymer composites for quite a long time. The entity of sandwiched layers, with $1 \mathrm{~nm}$ thickness and few hundred nanometers size in other two dimensions, is stacked together with van der Waals gap [2]. Depending on the space between clay platelets after incorporation with polymer matrix, the composites are roughly classified as following: firstly, exfoliated layers are fully open; secondly, intercalated layers are partially open [3].

Generally, incorporation of small amounts of filler can result in the exfoliated polymer composites form, which can greatly enhance mechanical antiflammable and heat-resistant properties as compared to original polymer matrix and intercalated dispersion form $[4,5]$. In order to overcome the interlayer van der Waals bonding barrier to achieve the layer exfoliation, in situ polymerization approach and melt intercalation method have been developed [6]. The catalyst for ethylene polymerization is introduced into the gallery of layered silicates after which polyethylene forms in situ and the layer structure delaminates [7]. This method has proved to be quite efficient in preparing exfoliated nanocomposites since the entropy resistance for the intercalation of polymer chains significantly reduces and the heat released by polymerization acts favorably to reduce the process-free energy [8].

The molecular architecture of polymers in the solid state is of fundamental importance to understand various macroscopic properties. Solid-state nuclear magnetic resonance (NMR) is one of the most informative techniques to characterize heterogeneous structures in polymer and polymer/clay nanocomposites [9]. Proton wide-line NMR and relaxation experiments have been widely applied to study the phase structure and chain dynamics. An intermediate component has been inferred from the data by fitting the ${ }^{1} \mathrm{H}$ wideline NMR spectra [10-12]. We [13] used fiber clay, named palygorskite (PLT), so as to immobilize metallocene catalyst. The exfoliated PE/palygorskite can be obtained after ethylene polymerization. ${ }^{1} \mathrm{H}$ solid-state NMR was used to quantitatively analyse the macromolecular dynamics and phase structure of the resulting polymer nanocomposites. VanderHart et al. $[14,15]$ used proton longitudinal relaxation time 
$\left(T_{1}\right)$ to estimate the dispersion degree of MMT. $T_{1}$ showed a pronounced dependence on the $\mathrm{Fe}^{3+}$ concentration due to the strong paramagnetic of the $\mathrm{Fe}^{3+}$. However, the ${ }^{1} \mathrm{H}$ NMR spectrum of solid polymers often exhibits severely broad signals due to the strong ${ }^{1} \mathrm{H}_{-}{ }^{1} \mathrm{H}$ dipolar coupling and random orientation of the molecule. Fast magic-angle spinning (MAS) has been successfully used to average the ${ }^{1} \mathrm{H}-{ }^{1} \mathrm{H}$ dipolar coupling and obtain high resolution spectra for organic solids [16]. Lee et al. [17] used proton MAS technique to study the interaction between proton and phosphoric acid in the poly(vinyl phosphoric acid). The results showed that hydrogen-bonded $\mathrm{P}-\mathrm{OH}$ could be used as proton conductor. Moreover, ${ }^{13} \mathrm{C}$ cross-polarization and magic-angle spinning (CP/MAS) NMR $[18,19]$ has been recognized as a powerful tool for investigating structures and dynamics of polymer composites. The combination of dipolar decoupling (DD), cross-polarization (CP), and MAS techniques permits one to detect chemical shifts and magnetic relaxation parameters that are directly related to the chain conformation and dynamics in each phase structure. Wang et al. [20] used solidstate ${ }^{13} \mathrm{C}-\mathrm{NMR}$ to study the conformational change and structural heterogeneity of surfactant molecules on the montmorillonite layer. Those surfactant molecules had higher chain mobility and disordered conformation. Moreover, the existence of two orthorhombic phases was also reported by Hillebrand et al. using solid-state ${ }^{13} \mathrm{C}-\mathrm{NMR}$ [21]. They found that the chains responsible for the orthorhombic crystalline phase with broad-line width made relative fast $180^{\circ}$ chain flips by a traveling chain twist defect already at room temperature. The orthorhombic crystalline phase with narrow-line width may indicate the perfect crystalline phase. Our latest work also used solid-state ${ }^{13} \mathrm{C}$-NMR to study the phase structure and chain dynamics of polyethylene/palygorskite obtained by in situ polymerization [22]. The results of ${ }^{13} \mathrm{C} C \mathrm{CP} / \mathrm{MAS}$ showed that the chain mobility of orthorhombic crystalline phase with broad resonance line is obviously hindered compared with the narrow resonance line phase when the filler is introduced.

In this work, the exfoliated and intercalated polyethylene/MMT nanocomposites were obtained according to the ethylene in situ polymerization, respectively. Subsequently, the phase structure and chain dynamics of nanocomposites with different dispersion state were investigated by solid-state NMR. ${ }^{1} \mathrm{H}$ and ${ }^{13} \mathrm{C}$ solid-state NMR was used to study phase composition and molecular mobility of the samples under static and MAS states. Finally, the change in phase structure of PE nanocomposites was compared based on the NMR results when MMT and PLT were introduced into PE matrix.

\section{Experiments}

2.1. Materials. Bis(cyclopentadienyl)titanium dichloride $\left(\mathrm{Cp}_{2} \mathrm{TiCl}_{2}\right.$, ACROS Organics) was purchased from $\mathrm{J} \& \mathrm{~K}$ Chemical (Shanghai, China) and used as received. Toluene was purified using Solvent Purification (Invention Tech. USA). Methylaluminoxane (MAO) in toluene (wt\%, 10\%) was purchased from J \& K Chemical (Shanghai, China).
Polymerization grade ethylene was obtained from Ningbo Gas Company (Ningbo, China) and purified through Gas Purification instrument (Shengmai Chemical Company, Dalian, China). $\left[\left(\mathrm{C}_{18} \mathrm{H}_{37}\right)_{2} \mathrm{~N}\left(\mathrm{CH}_{3}\right)_{2}\right]^{+} \mathrm{Cl}^{-}$and Ca-based MMT was purchased from J \& $\mathrm{K}$ Chemical. The content of $\mathrm{Fe}^{3+}$ of MMT was $3.1 \mathrm{wt} \%$ according to the product instrument.

\subsection{Preparation of Polyethylene and \\ Polyethylene/MMT Nanocomposites}

2.2.1. Preparation of the Modified MMT. The purification procedure of MMT was obtained as follows: $300 \mathrm{~g}$ MMT was dispersed in $5 \mathrm{~L}$ water and then stirred for 60 mins. $8.5 \mathrm{~g}$ $\mathrm{Na}_{2} \mathrm{CO}_{3}$ was added into the MMT suspension and stirred for $45 \mathrm{mins}$. The mixture was condensed for 120 mins. The supernatant was further rotated in ultracentrifugal instrument with $2000 \mathrm{rpm}$. Finally, the supernatant was dried at $90^{\circ} \mathrm{C}$ for $12 \mathrm{~h}$. The X-ray diffraction (XRD) results showed that interlayer spacing of purified MMT was $1.3 \mathrm{~nm}$.

$50 \mathrm{~g}$ purified MMT suspended in $6 \mathrm{~L}$ water. Then, $9.4 \mathrm{~g}$ $\left[\left(\mathrm{C}_{18} \mathrm{H}_{37}\right)_{2} \mathrm{~N}\left(\mathrm{CH}_{3}\right)_{2}\right]^{+} \mathrm{Cl}^{-}$was added into the mixture. The $\mathrm{pH}$ value of this mixture was maintained around 8.0 modulated by $\mathrm{HCl}$. Subsequently, the mixture was stirred for $120 \mathrm{~min}$ at $65^{\circ} \mathrm{C}$. The abundant $\mathrm{Cl}^{-}$was removed using distillated water. Finally O-MMT can be obtained after the mixture was dried. The XRD results showed that interlayer spacing of O-MMT was greatly increased to $3.8 \mathrm{~nm}$.

2.2.2. Ethylene Polymerization. The polyethylene bulk was prepared as follows. Ethylene polymerization was conducted in a $300 \mathrm{~mL}$ autoclave stainless steel reactor. The reactor was set to $80^{\circ} \mathrm{C}$ and purged five times by nitrogen and one time by ethylene before polymerization. Then the reactor temperature was set to $40^{\circ} \mathrm{C}$, and $200 \mathrm{~mL}$ toluene was poured into the reactor with cocatalyst $5 \mathrm{mmol}$ MAO. Ethylene pressure was fixed at $0.1 \mathrm{MPa}$. Polymerization started by

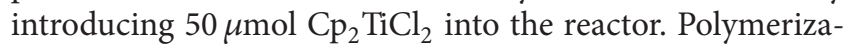
tion was then quenched by adding $20 \mathrm{~mL}$ acidified ethanol. Polymers are dried at $60^{\circ} \mathrm{C}$ under vacuum for $6 \mathrm{~h}$ after filtering. Polyethylene/MMT nanocomposites were prepared following the same method with pure polyethylene except that O-MMT was firstly introduced and stirred for $15 \mathrm{~min}$ under ultrasonic; then catalyst was introduced. The content of MMT in the polyethylene matrix was modulated according to the polymerization time.

\subsubsection{Characterization of the $P E$ and PE/MMT Samples.} Molecular weight (MW) and MW distribution (MWD) were determined by gel permeation chromatography (GPC) method at $150^{\circ} \mathrm{C}$ using a PL-GPC-220 (Polymer Laboratories, Darmstadt, Germany) instrument and 1,2,5-trichlorobenzene as solvents. Transmission electron micrographs (TEM) were obtained using a Phillips CM100 apparatus at an acceleration voltage of $100 \mathrm{kV}$. Each sample was prepared using an ultracryomicrotome cutting apparatus at $-130^{\circ} \mathrm{C}$, yielding samples $80 \mathrm{~nm}$ in thickness. A thermogravimetric analysis was carried out on a TGA/SDTA851 instrument 
(Me\ETTER Corp., Switzerland). The $\mathrm{N}_{2}$ flow rate was fixed at $20 \mathrm{~mL} \cdot \mathrm{min}^{-1}$. Heating took place from 30 to $1000^{\circ} \mathrm{C}$ at $10^{\circ} \mathrm{C} \cdot \mathrm{min}^{-1}$ heating rate. X-ray diffraction spectra of clay samples were recorded on a Rigaku D/Max-RA diffractometer (Japan) with $\mathrm{Cu} \mathrm{Ka}$ radiation $(40 \mathrm{KV}, 80 \mathrm{~mA})$. Scanning was done in $0.02^{\circ}$ steps at a speed of $2^{\circ} \cdot \min ^{-1}$.

2.3. NMR Measurements. ${ }^{1} \mathrm{H}$ solid-state NMR spectra, longitudinal magnetization relaxation $\left(T_{1}\right)$, and DQ build-up curves were obtained using a Bruker DSX-200 spectrometer at a proton frequency of $200.02 \mathrm{MHz}$. The data were collected for nonspinning samples. A $1.5 \mu \mathrm{s} 90^{\circ}$ pulse and a $5 \mathrm{~s}$ recycle delay were used for all experiments. The detailed experiment followed the same method with the former study [22]. ${ }^{13} \mathrm{C}$ solid-state NMR was carried out on Bruker DSX-500 spectrometer. The magic-angle spinning speed was $5 \mathrm{kHz}$ in all ${ }^{13} \mathrm{C} \mathrm{CP} / \mathrm{MAS}$ (cross-polarization and magic-angle spinning) and ${ }^{13} \mathrm{C}$ DP/MAS (direct-polarization and magic-angle spinning) NMR measurements using $2.5 \mathrm{~mm}$ MAS probe. ${ }^{13} \mathrm{C} \mathrm{CP} / \mathrm{MAS}$ were recorded using $5 \mathrm{~s}$ recycle time with changing the contact time.

\section{Results and Discussion}

3.1. Basic Information of the Polyethylene and Polyethylene/MMT Nanocomposites. The results of ethylene polymerization are shown in Table 1. Catalyst activity obviously decreases when more O-MMT is introduced. Prticularly, catalyst activity reaches to almost $25 \%$ of that in homogeneous catalyst when the loading of O-MMT is $7.2 \%$. It indicates that the electronegativity of the MMT greatly influences the catalyst activity although it is covered by $\left[\left(\mathrm{C}_{18} \mathrm{H}_{37}\right)_{2} \mathrm{~N}\left(\mathrm{CH}_{3}\right)_{2}\right]^{+} \mathrm{Cl}^{-}$layer. The defect of the $\left[\left(\mathrm{C}_{18} \mathrm{H}_{37}\right)_{2} \mathrm{~N}\left(\mathrm{CH}_{3}\right)_{2}\right]^{+} \mathrm{Cl}^{-}$cover layer, particularly on the side of MMT, causes the uncovered acid MMT layers contacting with catalyst active sites and thus decreases the activity [23].

Figures 1(a) and 1(b) compare the XRD results of PE and PE/MMT nanocomposites. The diffraction peak 001 of O-MMT disappears in the PEMT18, which indicates that the ordered structure of MMT is destroyed after ethylene polymerization. The distance between MMT layers is larger than $8 \mathrm{~nm}$ in the PEMT18 matrix. However, two diffraction peaks can be found in the small angle range of XRD spectra of PEMT71. The layer distance can be calculated as $5.07 \mathrm{~nm}$ and $1 \mathrm{~nm}$, respectively. This indicates that the MMT layer distance is smaller than PEMT18 and part of MMT layer may aggregate. The dispersion state of MMT in the polyethylene matrix is further investigated by TEM (Figures 1(c) and 1(d)). TEM morphology shows that the distance of MMT layer in PEMT18 is large and exfoliated nanocomposite is obtained. Moreover, MMT layer shows obviously aggregation behavior in PEMT71, and the intercalated dispersion is finally achieved.

\subsection{Solid-State NMR Experiments}

3.2.1. Phase Composition and Chain Dynamics by Proton NMR Spectra. Wide-line proton NMR bases its analysis on the extent of constrained molecular motions in the different domains [12]. The experimental wide-line spectra are deconvoluted into three components using the WinFit program. The broad component of the spectrum is approximated by a Gaussian function. This is a rough approximation of the actual line shape as shown in the following. The shape parameter $(\alpha=0.5)$ of the line with intermediate width (i.e., the interface) is between that of a Gaussian $(\alpha=1)$ and a Lorentzian $(\alpha=0)$ [22]. The differences in the three component line shapes are expected to arise from differences in polymer chain motilities. The crystalline chains will result in the broad line due to only a partial motional averaging of the dipole-dipole interaction between the protons along the chains.

Figure 2 shows the fitting results of proton wide-line NMR spectra measured at different temperature. Figure 2(a) shows large changes in phase composition when the MMT clay is introduced. As compared to the content of crystalline phase (crystallinity) in the bulk PE, the introduction of MMT into the PE matrix greatly increases the crystallinity in a range of temperature from 300 to $360 \mathrm{~K}$. It suggests that MMT clay layers interfere with the chain folding process leading to an increased content of well-ordered structures. Higher MMT amount in the polymer matrix also shows higher crystallinity. Interestingly, this is an opposite result compared with our former studies where the crystallinity was decreased as the palygorskite (PLT) loading increases in the PE/PLT nanocomposites [22]. In our opinion, the opposite dependence of crystallinity on filler loading may be attributed to the different structure of filler. MMT filler is the layer structure, while PLT filler presents the fiber style. Layer structure of MMT is able to adsorb more molecular chains on the surface compared with the fiber structure $[22,24]$. Moreover, these adsorbed chains contribute to more mobility confined chains which can result in higher crystallinity. This can also be inferred from the results of chain mobility in Figure 2(b). All the widths of three phases in the nanocomposites are larger than PE matrix at all measured temperatures which indicates that the chain mobility of nanocomposites is hindered.

\subsubsection{Chain Dynamics by Proton Double-Quantum Dipolar} Filter ( $\left.{ }^{1} H D Q N M R\right)$. The magnetization from the more rigid part of a heterogeneous polymer can be selected using a dipolar filter based on the excitation of the double-quantum coherences [25]. The efficiency of the DQ filter is measured for PE and PE/MMT samples in Figure 3. At small excitation times, the filter selects the magnetisation mainly from the crystalline regions (rigid part). At longer excitation times, the filter starts to act like a transverse relaxation time $\left(T_{2}\right)$ filter, and the magnetisation from the less-mobile and mobile amorphous regions can be selected. The faster chain motions in the intermediate and amorphous phases reduce the effectiveness of proton dipole-dipole interactions through motional averaging [11]. Thus, the absence of DQ signal indicates that the dipole-dipole coupling between protons is weak due to either large distance between proton pairs or fast molecular motion on an NMR time scale $(10-100 \mu \mathrm{s})$. 
TABLE 1: Ethylene polymerization in the presence of O-MMT and the characterization results of PE/MMT hybrid materials ${ }^{\mathrm{a}}$.

\begin{tabular}{cccccccc}
\hline Run & $\begin{array}{c}\text { MMT Conc. in feed } \\
\mathrm{g} \cdot \mathrm{L}^{-1}\end{array}$ & $\begin{array}{c}\text { Polymer yield } \\
\mathrm{g}\end{array}$ & $\begin{array}{c}\text { Activity } \\
\mathrm{Kg} \mathrm{PE} / \mathrm{mol}_{\mathrm{Cat}} \cdot \mathrm{h}\end{array}$ & $\begin{array}{c}\mathrm{Mw} \\
10^{5} \mathrm{~g} \cdot \mathrm{mol}^{-1}\end{array}$ & $\begin{array}{c}\text { Mw/Mn } \\
\text { MMT in PE }^{\mathrm{b}} \\
\text { wt.\% }\end{array}$ & $\begin{array}{c}\text { Sample } \\
\text { notation }\end{array}$ \\
\hline $1 \#$ & 0 & 22.0 & 2200 & 4.81 & 3.3 & 0 & PE \\
$2 \#$ & 0.50 & 10.9 & 1090 & 2.19 & 3.5 & 1.8 & PEMT18 \\
$3 \#$ & 0.45 & 5.9 & 590 & 1.37 & 2.9 & 7.1 & PEMT71 \\
\hline
\end{tabular}

a Polymerization conditions: solvent $400 \mathrm{~mL}$ toluene. Ethylene pressure $0.1 \mathrm{MPa}$. Cat. conc. In the feed $25 \mu \mathrm{mol} \mathrm{Ti} \cdot \mathrm{L}^{-1}$. Polymerization time $60 \mathrm{mins}$.

${ }^{\mathrm{b}} \mathrm{MMT}$ content in the polyethylene matrix was measured by TGA.

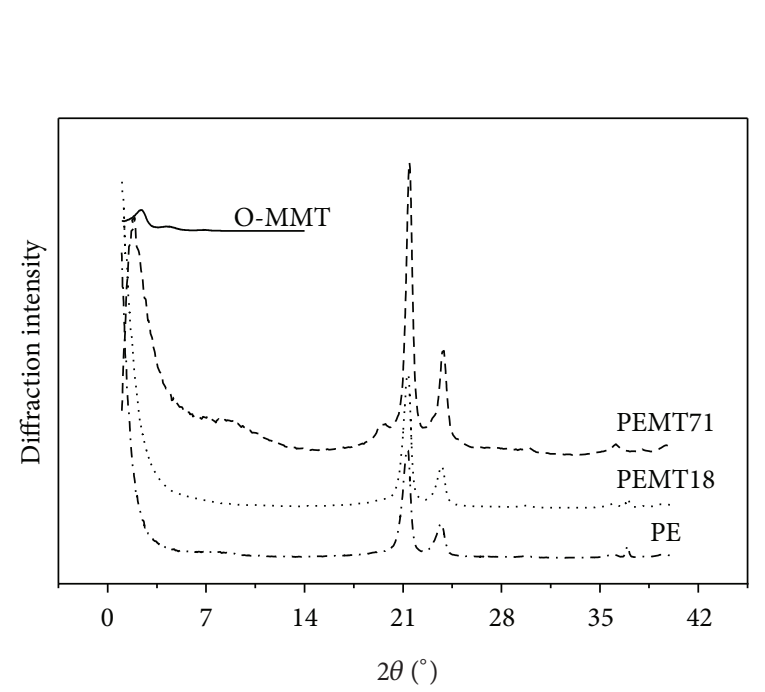

(a)

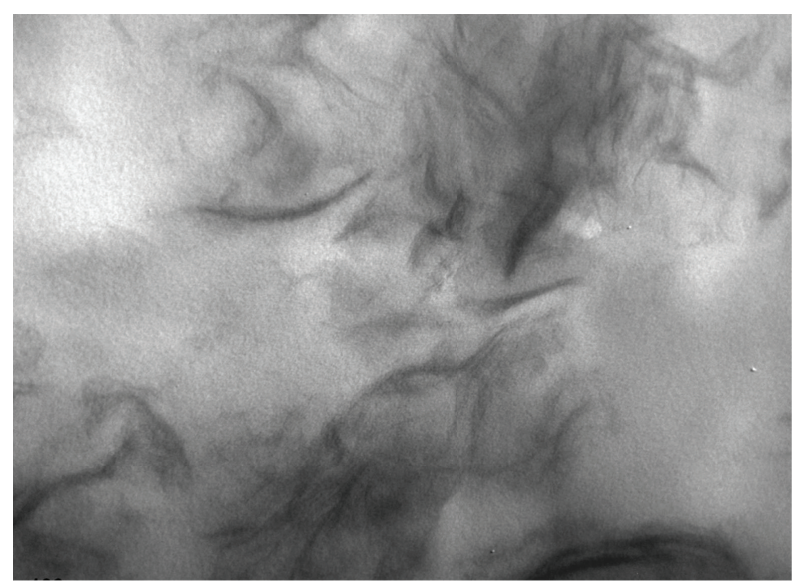

(c)

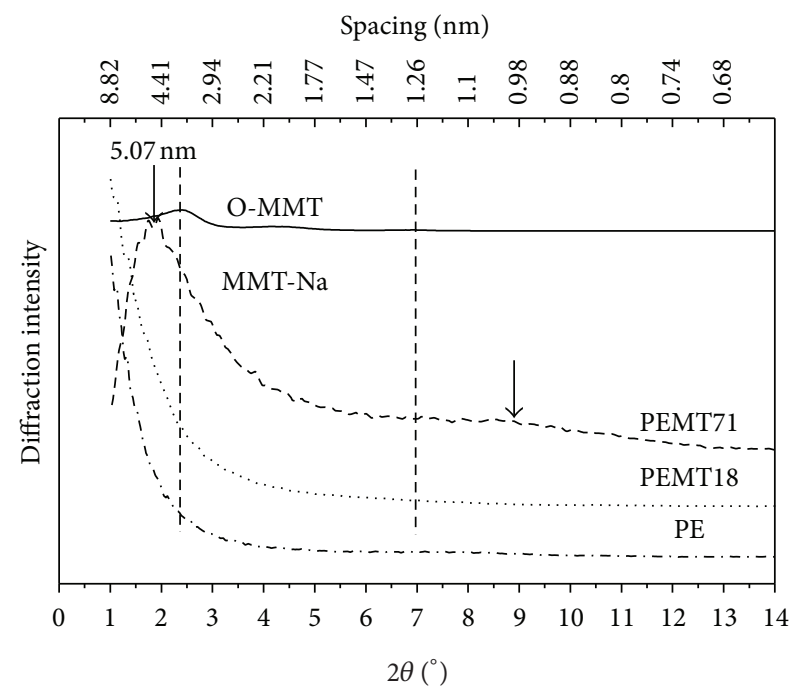

(b)

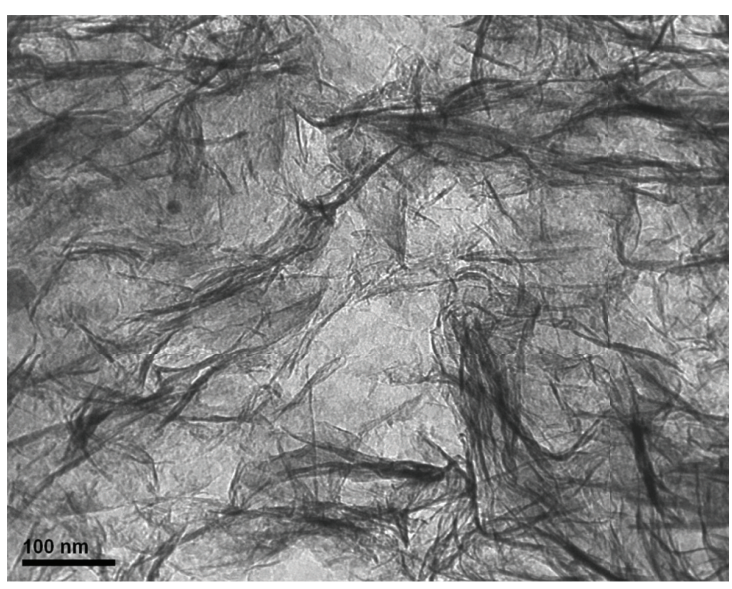

(d)

FIGURE 1: X-ray diffraction spectra (a, b) and TEM morphology (c, d) of PE and PE/MMT nanocomposites: (a) spectra in the full diffraction angle; (b) spectra in the small angle rang; (c) TEM morphology of PEMT18, ×200000; (d) TEM morphology of PEMT71, ×200000.

Figure 3(a) shows the normalized DQ build-up curves of samples measured at room temperature. The intensity of polyethylene matrix DQ spectrum is obviously lower than that of nanocomposites, suggesting that the nanocomposites are more rigid than the bulk PE. Moreover, the maxima of the curves appear at very short excitation time, in the range from 8 to 15 us, indicating the presence of strong ${ }^{1} \mathrm{H}$ dipolar interaction. This interaction represents the feature of crystalline phase in the PE samples. In our case, this interaction in nanocomposites is higher than that in the PE bulk and is enhanced in higher filler loading samples. It indicates that the chains of crystalline phase are more rigid in polymer nanocomposites, and the rigid degree increases when the filler loading goes up. Moreover, the initial stage of DQ build-up curves without normalization always presents linear shape. The slope of the initial curves can reflect the position of first maximum on the DQ curves. The maximum position will be delayed with a low slope indicating a more 


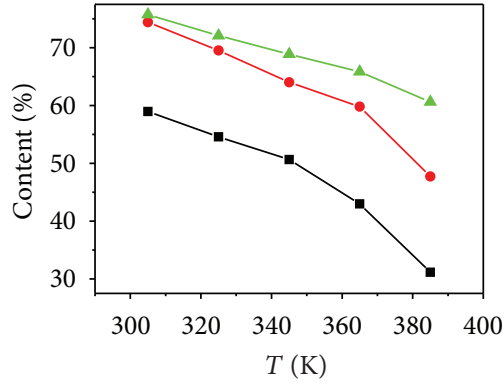

Crystalline phase

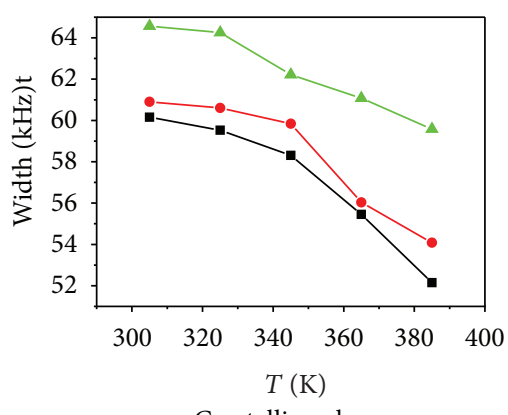

Crystalline phase

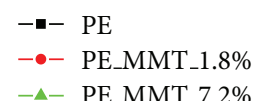

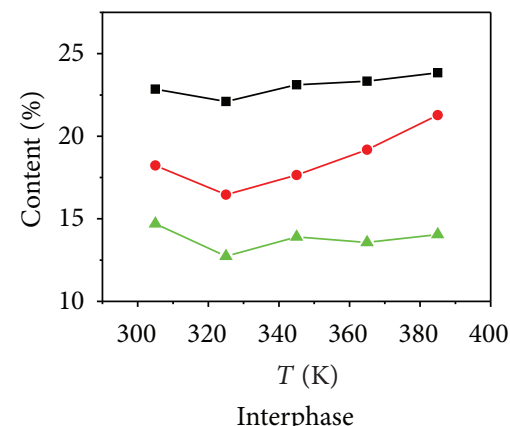

(a)

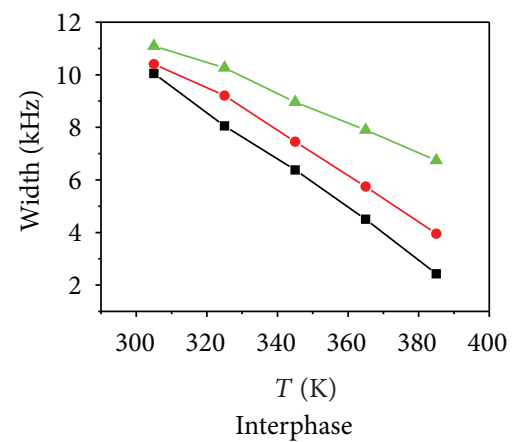

$-\square-P E$

- - PE_MMT_1.8\%

- - PE_MMT_7.2\%

(b)

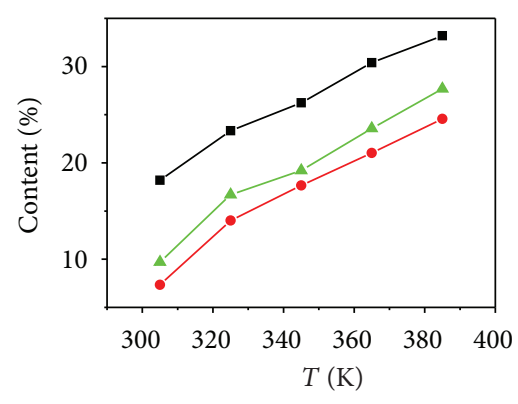

Amorphous phase

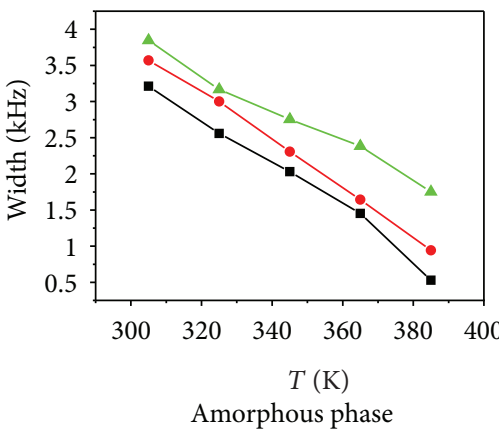

$-\mathbf{- P E}$

-•- PE_MMT_1.8\%

- $\triangle$ - PE_MMT_7.2\%

Figure 2: Fitting results of ${ }^{1} \mathrm{H}$ wide-line NMR spectra. (a) Phase composition and (b) line width.
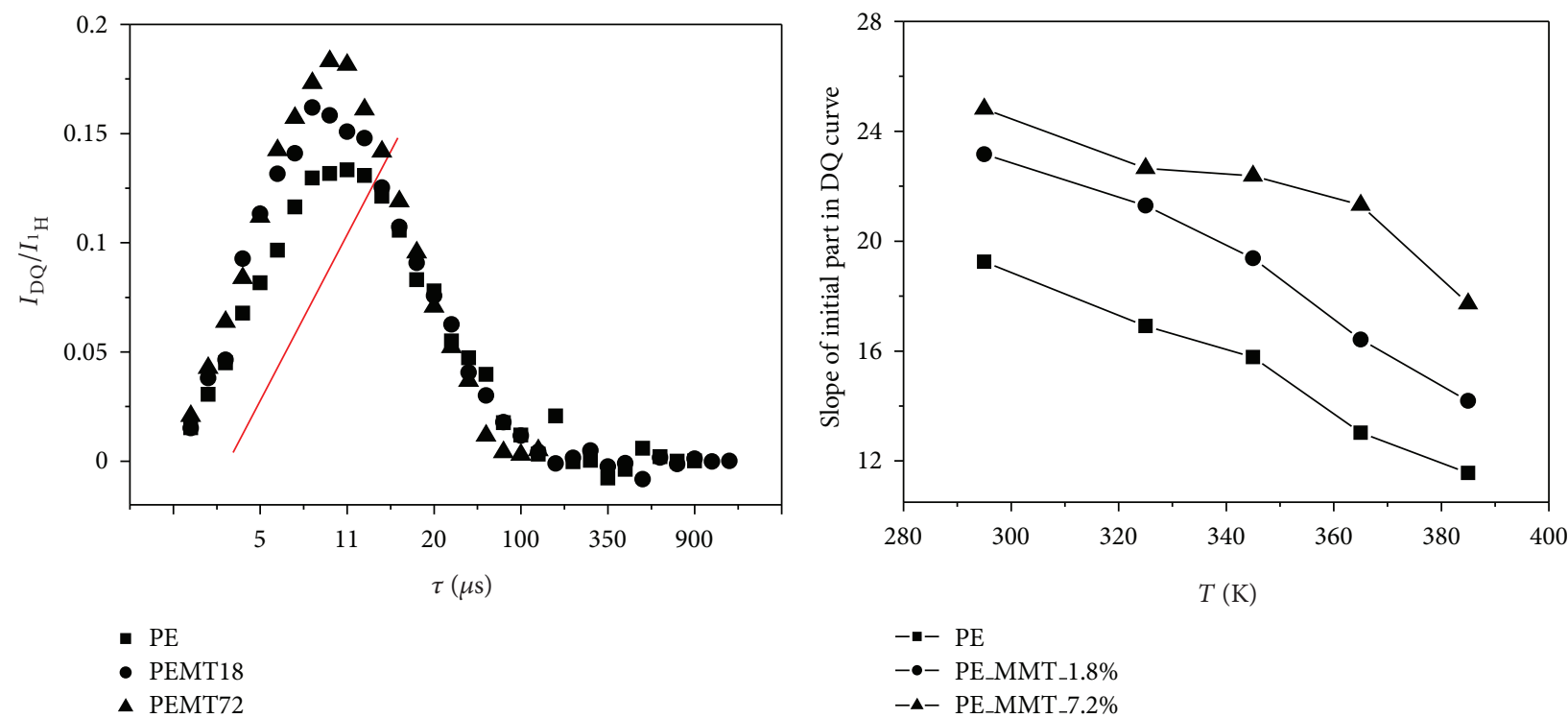

- PE

-•- PE_MMT_1.8\%

- PEMT72

(b)

FIGURE 3: The DQ built-up curves of PE and PE nanocomposites measured at room temperature. (a) DQ built-up curves and (b) the slope of initial part in DQ curve. 


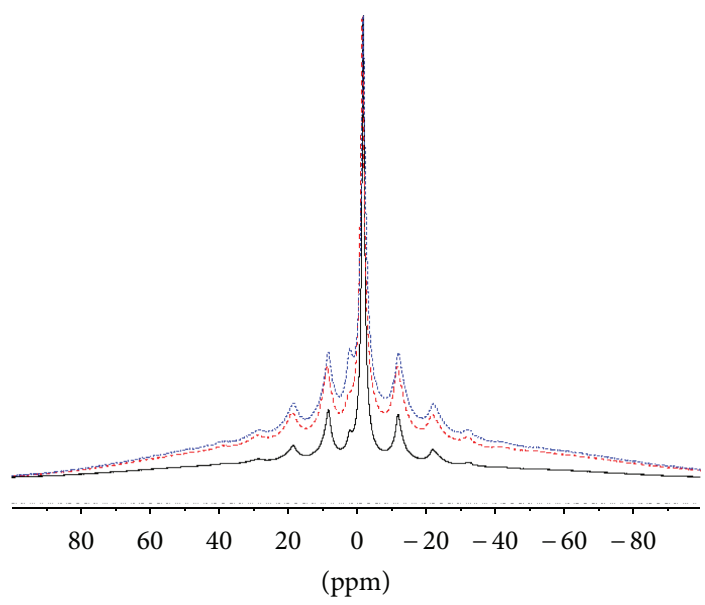

$(\mathrm{ppm})$

(a)

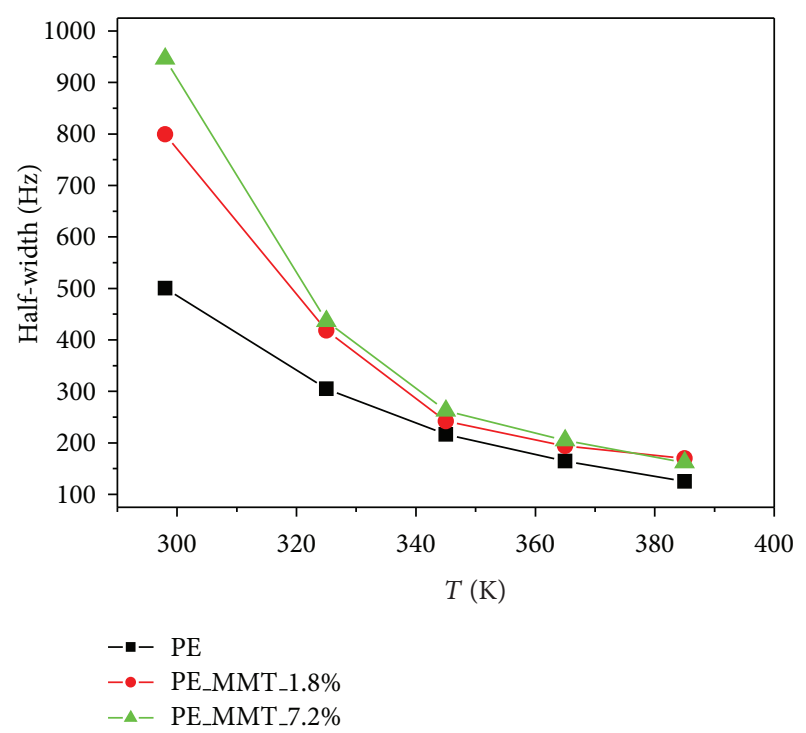

(b)

FIGURE 4: ${ }^{1} \mathrm{H} / \mathrm{MAS}$ spectrum of PE and PE nanocomposites. (a) A typical ${ }^{1} \mathrm{H} / \mathrm{MAS}$ spectrum measured at room temperature; (b) half width of the ${ }^{1} \mathrm{H}$ MAS spectra dependence on the temperature.

mobile phase [26]. Temperature-dependent measurements of the DQ build-up curves show some interesting features. The slope of initial stage of DQ curves measured at different temperature is shown in Figure 3(b). The slope decreases upon increasing the temperature. It indicates that the chain mobility of crystalline phase is enhanced. At all temperatures, the slope of nanocomposites is obviously higher than that of polyethylene bulk and also increases as the MMT loading increased. It suggests that the chains become more rigid in crystalline phase when the MMT load is increased. Thus, the results of DQ build-up curves show good correspondence with those of wide-line proton NMR.

3.2.3. Chain Dynamics by ${ }^{1} H$ MAS Spectrum. Solid-state variable-temperature/magic-angle spinning (MAS) ${ }^{1} \mathrm{H}$ NMR measurements are carried out. The results are shown in Figure 4. For semicrystalline polyethylene, the line width of the ${ }^{1} \mathrm{H}$ signal is dominated by the dipolar interactions between protons. The magnitude of such interaction is usually several tens of $\mathrm{kHz}$, and it is possible to achieve high resolution in the ${ }^{1} \mathrm{H}$ spectrum by MAS at a speed of $5 \mathrm{kHz}$ in our experiments. During MAS process, strong dipolar couplings from the restricted motions of molecules can be removed. Thus, the soft phase information where the chains mobility has more freedom can be reflected from the proton MAS spectrum [16]. In our results, the half width of PE nanocomposites spectrum is higher than that of PE bulk at all measurements, which indicates that the chains in soft phases are more rigid due to the addition of MMT filler.

Moreover, the difference of line width in PEMT18 and PEMT71 gradually disappears upon increasing the temperature (Figure 4(b)), while the line width of these samples are almost the same at $365 \mathrm{~K}$ and $385 \mathrm{~K}$ where the temperature is near to the melting point. This may indicate that the MMT loading plays little effect on the chain dynamics to the chains in soft phases when the chain crystallinity reaches to melting state. However, it needs to be further proved.

\subsubsection{Chain Dynamics by ${ }^{1} H$ Longitudinal Magnetization} Relaxation $\left(T_{1}\right)$. Less numerous paramagnetic $\mathrm{Fe}^{3+}$ ions exist in the MMT with a concentration of $3.1 \mathrm{wt} \%$. However, these ions are strongly paramagnetic and can exert an influence on the nuclear spins near the clay surface. Zhang et al. [27] described the impact of $\mathrm{Fe}^{3+}$ ions on $\mathrm{Li}^{+}$spins in intercalated MMT clays. The paramagnetic impurities had a great influence on the spin-lattice relaxation time of the protons. The longitudinal magnetization relaxation $\left(T_{1}\right)$ decreased when the paramagnetic influence occurred.

The recovery of the magnetization is well fitted with a sum of two components, and the results are shown in Figure 5. Higher values of relaxation times are observed when $1.8 \mathrm{wt} \%$ MMT introduces into the bulk polyethylene. It suggests that the presence of the filler leads to an immobilization of the chain in rigid phase (Figure 5(a)) and soft phase (Figure 5(b)). This effect is in agreement with the findings from the wide-line NMR. However, the relaxation time is obviously decreased when the filler loading increases to $7.1 \mathrm{wt} \%$. The influence of paramagnetic of $\mathrm{Fe}^{3+}$ ions occurs due to the intercalated dispersion state of PEMT71. It is believed that the $\mathrm{Fe}^{3+}$ mainly resides in the octahedral sites of MMT and can be enriched on the outside of the clay layers [2]. The protons of the organic modifier $\left(\left[\left(\mathrm{C}_{18} \mathrm{H}_{37}\right)_{2} \mathrm{~N}\left(\mathrm{CH}_{3}\right)_{2}\right]^{+} \mathrm{Cl}^{-}\right)$on the clay can become as close as $0.5-0.6 \mathrm{~nm}$ from the $\mathrm{Fe}^{3+}$ ions. Such protons, as well as certain $\mathrm{PE}$ protons that are very proximate to the surface, will experience both a direct line broadening and a shortening of $T_{1}[14,15]$. The layers between MMT are closer in the intercalated dispersion state. The influence 


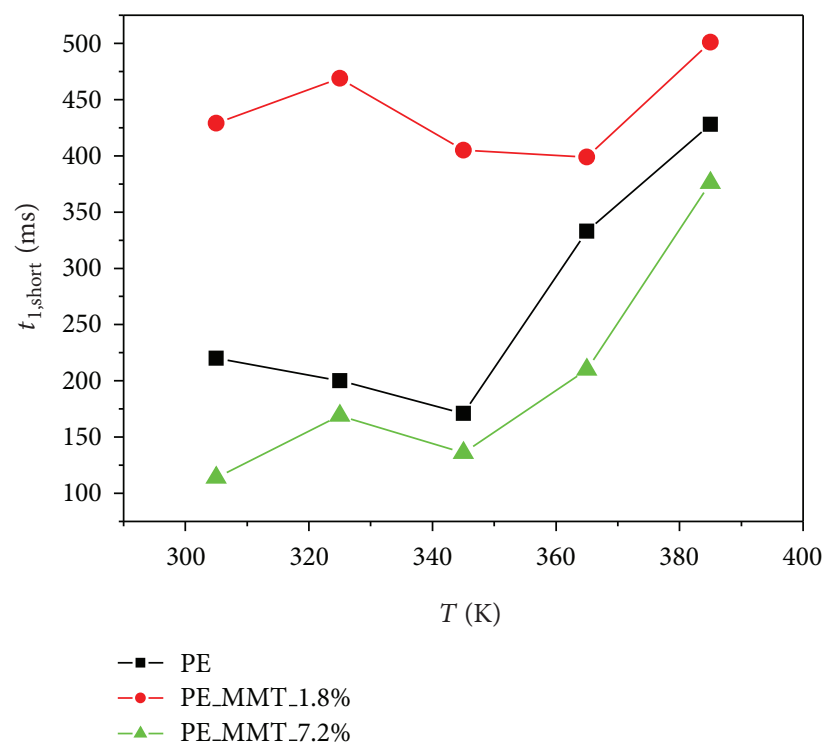

(a)

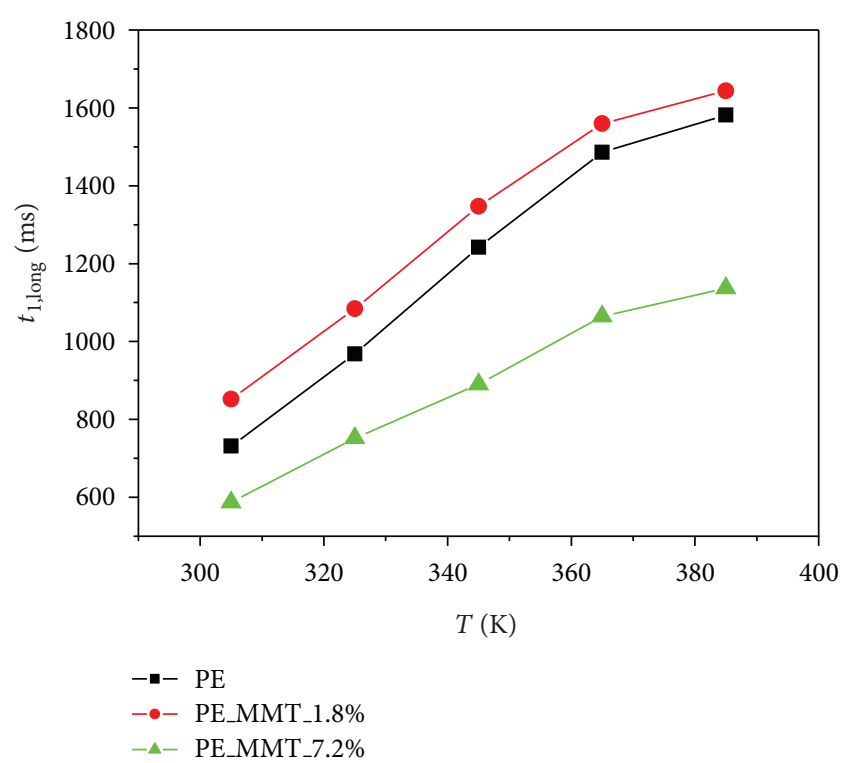

(b)

FIGURE 5: Longitudinal magnetization relaxation $T_{1}$ value dependence on the temperature.

of $\mathrm{Fe}^{3+}$ on the relaxation time will be enhanced compared with a relative larger distance of MMT in exfoliated PE/MMT nanocomposites.

\subsubsection{Phase Composition and Chain Dynamics by ${ }^{13} \mathrm{C}$ Solid-}

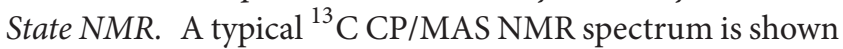
in Figure 6. The spectrum is fully deconvoluted by Lorentizian line shapes. A combination of four components fitting leads to the best fitting results for the spectrum measured at RT. The four components correspond to the different phases: amorphous phase $(30.82 \mathrm{ppm})$, intermediate phase (31.64 ppm), orthorhombic crystalline phase $(32.67 \mathrm{ppm})$, and monoclinic phase (33.74 ppm). Based on the gauche effect, ${ }^{13} \mathrm{C}$ NMR can distinguish between orthorhombic and amorphous phases. For the former, segments are in alltrans conformation, and ${ }^{13} \mathrm{C}$ NMR resonance appears at $32.9 \mathrm{ppm}$. For the latter, the $30.8 \mathrm{ppm}$ line is from gauche containing conformation [27]. Moreover, polyethylene usually takes two types of crystal forms: the stable orthorhombic and metastable monoclinic phases. The orthorhombic phase occurs under normal conditions, and the latter appears after straining the PE [20]. The chains in these two phases take all zig-zag conformation but pack in different ways. The planes of the all-trans chains in the monoclinic phase are parallel to one another. However, they are perpendicular to each other in the orthorhombic phase.

Recently, much attention has been paid to deal with the existence of the third crystalline phase [21]. Our latest publication also detected out two orthorhombic crystalline phase in the PE/PLT nanocomposites by ${ }^{13} \mathrm{C} \mathrm{CP} / \mathrm{MAS}$ NMR [22]. The chains responsible for the $\mathrm{Cr}-\mathrm{b}$ carbon resonance, which can form the main fraction of the crystalline domains, make relatively fast $180^{\circ}$ chain flips by a traveling chain twist defect already at room temperature. Thus, this resonance is

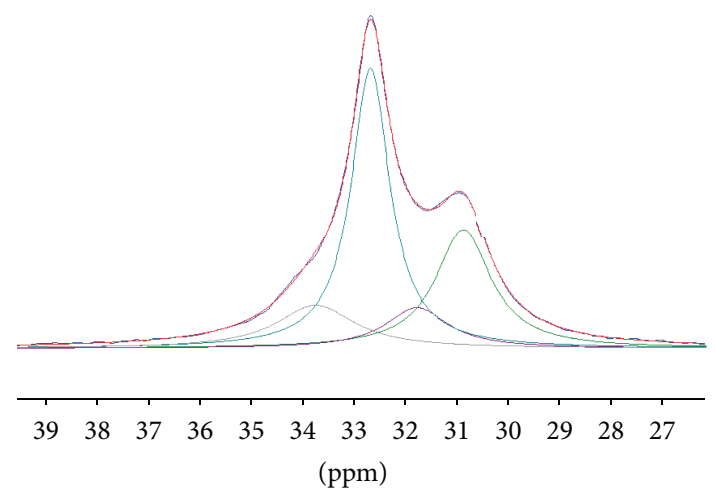

Figure 6: Fitting results of ${ }^{13} \mathrm{C} \mathrm{CP} / \mathrm{MAS}$ of PE spectra when the CP time is $1 \mathrm{~ms}$ at RT.

associated with the defective crystalline phase. The chains responsible for the $\mathrm{Cr}-\mathrm{n}$ carbon resonance do not show these motions and probably are found in more perfect crystalline structures. However, only one orthorhombic phase with narrow-line width is found in PE/MMT nanocomposites, indicating that more perfect crystalline phase is obtained in PE/MMT nanocomposites.

The crystallinity cannot be calculated directly from the ${ }^{13} \mathrm{C}$ CP/MAS spectrum, because of the different crosspolarization efficiency of crystalline and amorphous phase. DP/MAS ${ }^{13} \mathrm{C}$ spectrum is required to measure the crystallinity [20]. The results are listed in Table 2. The contents of orthorhombic phase and monoclinic phase in PE/MMT nanocomposites are both higher than those in polyethylene bulk at room temperature. This shows good correspondence with the results obtained by proton NMR. The line widths of amorphous phase and interphase in nanocomposites 

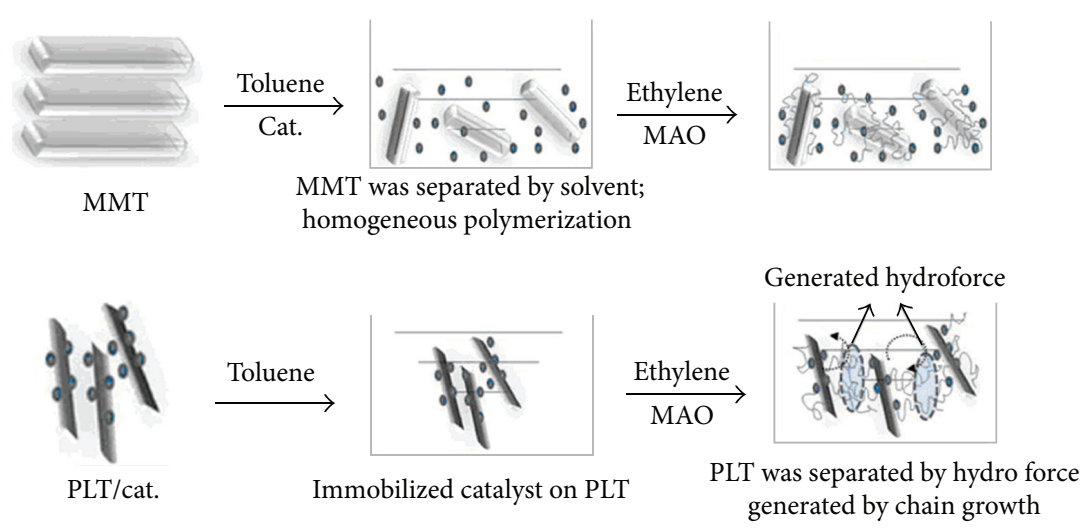

FIgURE 7: Proposed scheme of different polymerization methods for preparing nanocomposites.

TABLE 2: Results of ${ }^{13} \mathrm{CDP} / \mathrm{MAS}$ experiment.

\begin{tabular}{cccccccccc}
\hline & \multicolumn{4}{c}{ Phase Composition \% } & \multicolumn{5}{c}{ Half-width Hz } \\
\hline & CS (ppm) & $30.82 \pm 0.01$ & $31.64 \pm 0.01$ & $32.67 \pm 0.01$ & $33.74 \pm 0.01$ & $30.82 \pm 0.01$ & $31.64 \pm 0.01$ & $32.67 \pm 0.01$ & $33.74 \pm 0.01$ \\
RT & $0 \%$ & 59.83 & 4.45 & 29.35 & 6.37 & 145.9 & 104.6 & 119.4 & 174.4 \\
& $1.8 \%$ & 40.66 & 11.42 & 38.3 & 9.62 & 151.4 & 138.5 & 110.2 & 172.4 \\
& $7.2 \%$ & 38.34 & 11.13 & 34.53 & 15.97 & 173.5 & 198.8 & 98.1 & 280.9 \\
\hline & CS (ppm) & $29.77 \pm 0.01$ & $30.44 \pm 0.01$ & $32.25 \pm 0.01$ & $33.76 \pm 0.01$ & $29.77 \pm 0.01$ & $30.44 \pm 0.01$ & $32.25 \pm 0.01$ & $33.76 \pm 0.01$ \\
$385 \mathrm{~K}$ & $0 \%$ & 77.99 & 3.63 & 14.25 & 4.13 & 45.7 & 162.2 & 153.3 & 327.5 \\
& $1.8 \%$ & 75.24 & 6.55 & 16.41 & 0.9 & 60.2 & 178.1 & 139.3 & 127.2 \\
& $7.2 \%$ & 71.99 & 10.81 & 17.16 & - & 51.5 & 326.5 & 136.3 & - \\
\hline
\end{tabular}

are both higher than those of polyethylene bulk. The line width also broadens as increasing the filler loading, which means that the chain mobility in these two phases is more rigid. Furthermore, the line width of orthorhombic phase in nanocomposite gradually decreases compared with polyethylene bulk, while the line width of monoclinic phase obviously increases. It indicates that the introduction of MMT has more influences on monoclinic crystalline phase. The proton dipolar-dipolar interaction from monoclinic crystalline phase may contribute more in broadening the proton wideline NMR spectrum. The monoclinic phase of PE/MMT nanocomposites almost disappears when the temperature reaches to the melting point. It suggests that the monoclinic phase is more sensitive to the temperature compared to the orthorhombic phase due to its metastable state.

3.2.6. The Influence of Filler Style on the Crystalline Behaviors of Nanocomposites. In summary, chain dynamics and phase composition investigated by solid-state NMR show that MMTs with layer structure have obviously different influence on polyethylene crystalline behaviors compared with the PLT with fiber style, especially in crystalline phase. The reasons may be concluded as follows. first, the surface area of MMT is larger than fiber PLT filler due to the 2D layer structure of MMT [20]. Larger surface area can adsorb more polymer chains during the polymerization. This can result in much interaction between the polymer chains; thus more rigid chains will be obtained. Secondly, the polymerization method of PE/PLT and PE/MMT is a bit different which is shown in Figure 7. $\mathrm{Cp}_{2} \mathrm{TiCl}_{2}$ was firstly supported on the outer surface of PLT fibers during the preparation of PE/PLT nanocomposites. Polymerization directly occurred on the surface of PLT. The crystalline behavior of those growing chains was influenced as soon as the chain growth starts $[28,29]$. The physical structure and surface chemistry of PLT could both influence the nascent growing chains. Thus, this results in more defective crystalline phase. However, the chain growing process of PE/MMT is different. MMT filler is a multilayer structure. The distance between each layer is about $1.24 \mathrm{~nm}$ in the original MMT. This distance mainly increases to $3.68 \mathrm{~nm}$ after being modified by $\left[\left(\mathrm{C}_{18} \mathrm{H}_{37}\right)_{2} \mathrm{~N}\left(\mathrm{CH}_{3}\right)_{2}\right]^{+} \mathrm{Cl}^{-}$. The modified MMT (O-MMT) can achieve best dispersion in toluene after ultrasonic 15 mins. Polymerization was conducted by $\mathrm{Cp}_{2} \mathrm{TiCl}_{2} /$ toluene solution after the best dispersion state was obtained. Here, the polymerization was actually conducted by homogeneous $\mathrm{Cp}_{2} \mathrm{TiCl}_{2}$. The tensile force coming from the chain growth could easily separate MMT layers without conquering the layer force between them (support fragmentation). Thus, the effect of support fragmentation to the chain folder process was less [28]. MMT can mainly act as the nucleator when the growing chain reaches to a relative large scale. The great influence on crystalline behavior of the growing chains may arrive from the solution. As a result, more confined molecular chains can be obtained.

\section{Conclusion}

In this work, solid-state NMR is used to study the phase structure and chain dynamics in the exfoliated and intercalated 
PE/MMT nanocomposites prepared by in situ polymerization. A combination of proton and carbon methods under static and MAS conditions are used for the detailed study of phase structure. The results show that the incorporation of MMT with layer structure causes a large change in the phase structure. This structure also shows great difference from that of PE/PLT nanocomposites. The incorporated MMT enhances the crystallization process which results in higher crystallinity in PE/MMT nanocomposites. The polyethylene chains in the crystalline phase, interphase, and amorphous phase are also hindered due to the addition of MMT. ${ }^{13} \mathrm{C}$ $\mathrm{CP} / \mathrm{MAS}$ NMR shows that only one orthorhombic crystalline phase with narrow-line width can be detected. ${ }^{13} \mathrm{C}$ DP/MAS NMR are quantitatively used to measure the phase structure and chain dynamics. The results show that the introduction of MMT has more influences on monoclinic crystalline phase. The proton dipolar-dipolar interaction from monoclinic crystalline phase may contribute more in broadening the proton wide-line NMR spectrum. The monoclinic phase of PE/ MMT nanocomposites almost disappears when the temperature reaches to the melting point due to its metastable state.

\section{Conflict of Interests}

The authors declare that there is no conflict of interests. They have bought the materials from a company that has no relationship with this paper. This paper was only attributed to our research group.

\section{Acknowledgments}

Funding from the Project of Natural Science Foundation of China (no. 21206078), the Natural Science Foundation of Zhejiang Province (LQ12B06003), Zhejiang Province Department of Education Fund (Y201223797, Y201223742), K. C. Wong Education Foundation, and K. C. Wong Magna Fund, Hong Kong, are gratefully acknowledged. Professor Zhongren Chen's group is also acknowledged.

\section{References}

[1] M. Alexandre and P. Dubois, "Polymer-layered silicate nanocomposites: preparation, properties and uses of a new class of materials," Materials Science and Engineering R, vol. 28, no. 1-2, pp. 1-63, 2000.

[2] S. S. Ray and M. Okamoto, "Polymer/layered silicate nanocomposites: a review from preparation to processing," Progress in Polymer Science, vol. 28, no. 11, pp. 1539-1641, 2003.

[3] A. B. Morgan and J. W. Gilman, "Characterization of polymerlayered silicate (clay) nanocomposites by transmission electron microscopy and X-ray diffraction: a comparative study," Journal of Applied Polymer Science, vol. 87, no. 8, pp. 1329-1338, 2002.

[4] M. Tanniru, Q. Yuan, and R. D. K. Misra, "On significant retention of impact strength in clay-reinforced high-density polyethylene (HDPE) nanocomposites," Polymer, vol. 47, no. 6 , pp. 2133-2146, 2006.
[5] J. Zhang and C. A. Wilkie, "Polyethylene and polypropylene nanocomposites based on polymerically-modified clay containing alkylstyrene units," Polymer, vol. 47, no. 16, pp. 5736-5743, 2006.

[6] Q. H. Zeng, D. Z. Wang, A. B. Yu, and G. Q. Lu, "Synthesis of polymer-montmorillonite nanocomposites by in situ intercalative polymerization," Nanotechnology, vol. 13, no. 5, pp. 549-553, 2002.

[7] S. Ray, G. Galgali, A. Lele, and S. Sivaram, "In situ polymerization of ethylene with bis(imino)pyridine iron(II) catalysts supported on clay: the synthesis and characterization of polyethylene-clay nanocomposites," Journal of Polymer Science A, vol. 43, no. 2, pp. 304-318, 2005.

[8] M. Kawasumi, "The discovery of polymer-clay hybrids," Journal of Polymer Science A, vol. 42, no. 4, pp. 819-824, 2004.

[9] B. Blümich, NMR Imaging of Materials, Clarendon Press, Oxford, UK, 2000.

[10] D. E. Demco, G. Rata, R. Fechete, and B. Blümich, "Self-diffusion anisotropy of small penetrant molecules in deformed elastomers," Macromolecules, vol. 38, no. 13, pp. 5647-5653, 2005.

[11] A. Buda, D. E. Demco, M. Bertmer et al., "Domain sizes in heterogeneous polymers by spin diffusion using single-quantum and double-quantum dipolar filters," Solid State Nuclear Magnetic Resonance, vol. 24, no. 1, pp. 39-67, 2003.

[12] C. Hedesiu, D. E. Demco, R. Kleppinger et al., "The effect of temperature and annealing on the phase composition, molecular mobility and the thickness of domains in high-density polyethylene," Polymer, vol. 48, no. 3, pp. 763-777, 2007.

[13] W. Li, A. Adams, J. D. Wang, B. Blümich, and Y. R. Yang, "Polyethylene/palygorskite nanocomposites: preparation by in situ polymerization and their characterization," Polymer, vol. 51, no. 21, pp. 4686-4697, 2010.

[14] D. L. VanderHart, A. Asano, and J. W. Gilman, "Solid-state NMR investigation of paramagnetic nylon- 6 clay nanocomposites. 1. Crystallinity, morphology, and the direct influence of $\mathrm{Fe}^{3+}$ on nuclear spins," Chemistry of Materials, vol. 13, no. 10, pp. 3781-3795, 2001.

[15] D. L. VanderHart, A. Asano, and J. W. Gilman, "Solid-state NMR investigation of paramagnetic nylon-6 clay nanocomposites. 2. Measurement of clay dispersion, crystal stratification, and stability of organic modifiers," Chemistry of Materials, vol. 13, no. 10, pp. 3796-3809, 2001.

[16] Q. Chen, H. Hurosu, I. Ando, and X. Wu, "Solid-state variabletemperature $1 \mathrm{H}$ MAS NMR studies on deuterated polyethylene," Solid State Nuclear Magnetic Resonance, vol. 7, no. 4, pp. 319-325, 1997.

[17] Y. J. Lee, B. Bingöl, T. Murakhtina et al., "High-resolution solid-state NMR studies of poly(vinyl phosphonic acid) protonconducting polymer: molecular structure and proton dynamics," Journal of Physical Chemistry B, vol. 111, no. 33, pp. 9711-9721, 2007.

[18] F. A. Bovey and P. A. Mirau, NMR of Polymers, Academic Press, New York, NY, USA, 1996.

[19] K. Schmidt and H. W. Spiess, Multidimensional Solid-State NMR and Polymers, Academic Press, London, UK, 1994.

[20] L. Y. Wang, P. F. Fang, C. H. Ye, and J. W. Feng, "Solid-state NMR characterizations on phase structures and molecular dynamics of poly(ethylene-co-vinyl acetate)," Journal of Polymer Science $B$, vol. 44, no. 19, pp. 2864-2879, 2006. 
[21] L. Hillebrand, A. Schmidt, A. Bolz et al., "Nuclear magnetic resonance detection of two distinctly different chains in the orthorhombic crystalline phase of polyethylenes," Macromolecules, vol. 31, no. 15, pp. 5010-5021, 1998.

[22] W. Li, B. Jiang, A. Buda et al., "An NMR investigation on the phase structure and molecular mobility of the novel exfoliated polyethylene/palygorskite nanocomposites," Journal of Polymer Science B, vol. 48, no. 12, pp. 1363-1371, 2010.

[23] J. Heinemann, P. Reichert, R. Thomann, and R. Mülhaupt, "Polyolefin nanocomposites formed by melt compounding and transition metal catalyzed ethene homo- and copolymerization in the presence of layered silicates," Macromolecular Rapid Communications, vol. 20, no. 8, pp. 423-430, 1999.

[24] H. Sertchook, H. Elimelech, C. Makarov et al., "Composite particles of polyethylene @ silica," Journal of the American Chemical Society, vol. 129, no. 1, pp. 98-108, 2007.

[25] M. Bertmer, L. Gasper, D. E. Demco, B. Blümich, and V. M. Litvinov, "Investigation of soft component mobility in thermoplastic elastomers using homo- and heteronuclear dipolar filtered $1 \mathrm{H}$ double quantum NMR experiments," Macromolecular Chemistry and Physics, vol. 205, no. 1, pp. 83-94, 2004.

[26] M. Bertmer, M. Wang, D. E. Demco, and B. Blümich, "Segmental mobility in short-chain grafted-PDMS by homo- and heteronuclear residual dipolar couplings," Solid State Nuclear Magnetic Resonance, vol. 30, no. 1, pp. 45-54, 2006.

[27] Q. J. Zhang, W. X. Lin, G. Yang, and Q. Chen, "Studies on the phase structure of ethylene-vinyl acetate copolymers by solidstate ${ }^{1} \mathrm{H}$ and ${ }^{13} \mathrm{C}$ NMR," Journal of Polymer Science B, vol. 40, no. 19, pp. 2199-2207, 2002.

[28] T. F. L. Mckenna, A. D. Martino, G. Weickert, and J. B. P. Soares, "Particle growth during the polymerization of olefins on supported catalysts, 1-nascent polymer structures," Macromolecular Reaction Engineering, vol. 4, no. 1, pp. 40-64, 2010.

[29] D. Jauffrès, O. Lame, G. Vigier, and F. Doré, "How nascent structure of semicrystalline polymer powders enhances bulk mechanical properties," Macromolecules, vol. 41, no. 24, pp. 9793-9801, 2008. 

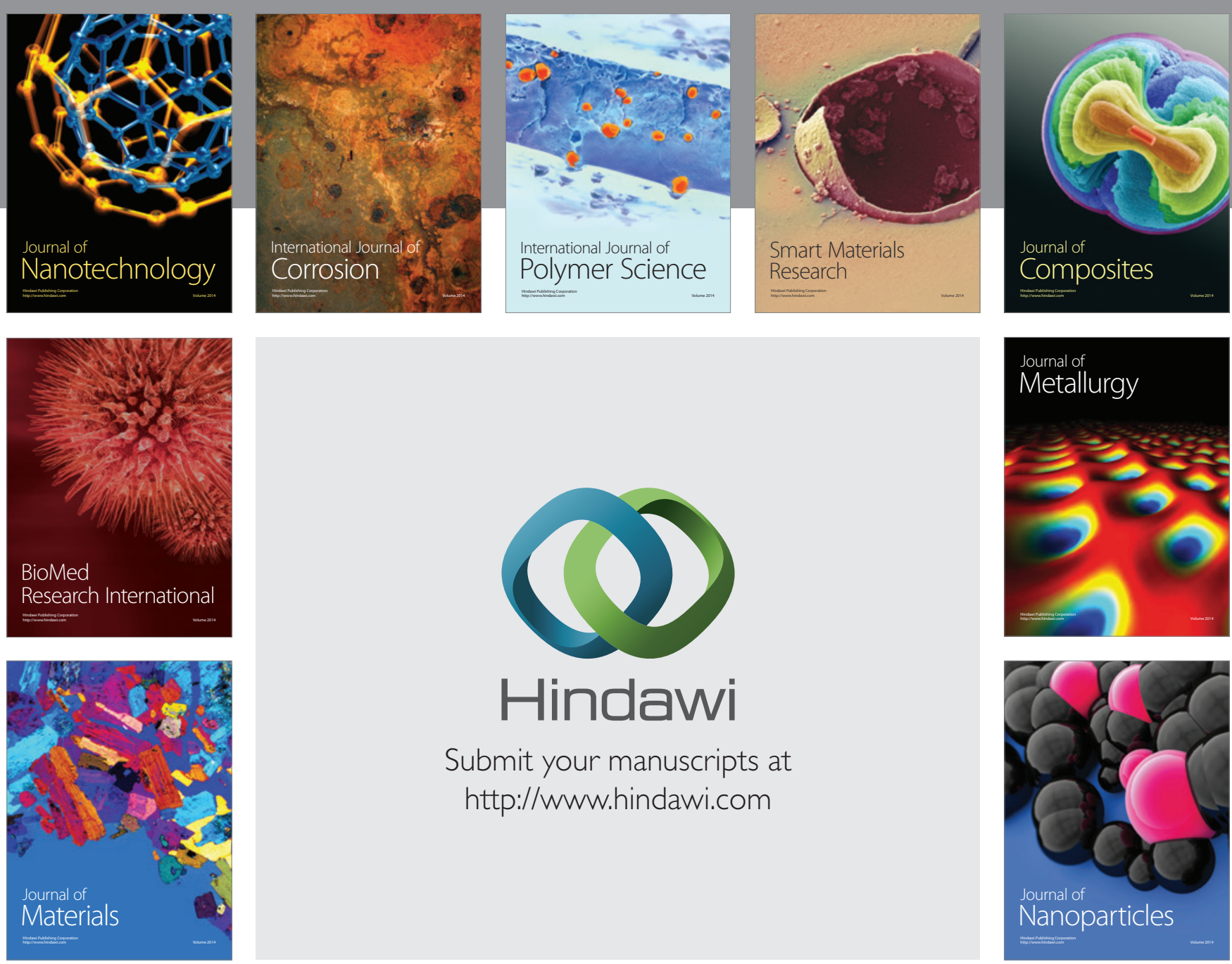

Submit your manuscripts at http://www.hindawi.com
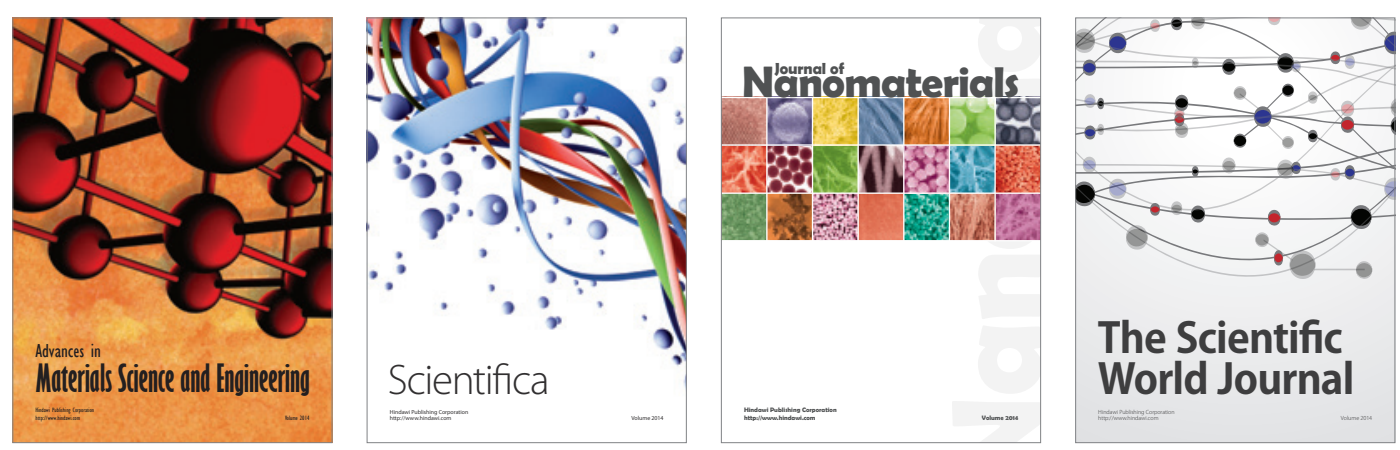

\section{The Scientific World Journal}
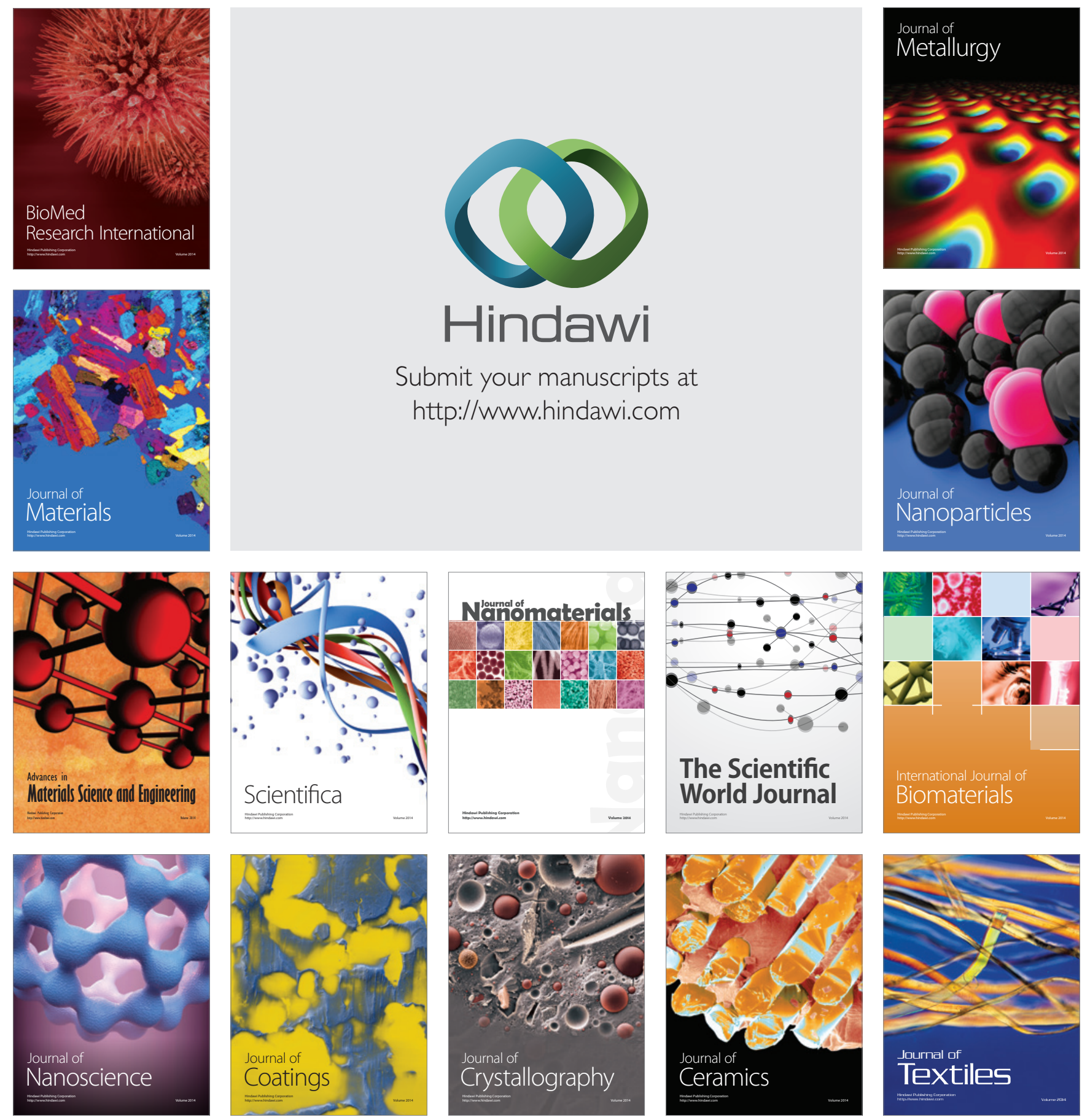